\title{
Apparent Failure of Automated Threshold Evaluation: What is the Mechanism?
}

\author{
Vikas Kalra, M.B.B.S. \\ Lynne Foreman, R.N. \\ Deepak Bhakta, M.D.
}

\section{Case Summary}

A 57-year-old man developed severe symptomatic tricuspid regurgitation requiring bioprosthetic valve replacement surgery utilizing St. Jude Medical Epic model \#E100-31M-00 (St. Paul, MN, USA) stented tissue valve. Complete atrioventricular (AV) block with junctional escape rhythm (ventricular rate 59-76 per minute) was noted during the postoperative period. Given clinical stability, observation with monitoring for return of $\mathrm{AV}$ conduction was recommended with hospital discharge occurring on postoperative day 7. Serial outpatient follow-up visits over the next 4 weeks demonstrated persistent AV block with junctional escape rhythm accompanied by symptoms of progressive exercise intolerance and energy loss, attributed to loss of AV synchrony. Six weeks after AV block was first detected, transvenous dual-chamber pacemaker implantation was performed using St. Jude Medical Allure Quadra RF PM3242, with active fixation atrial lead (St. Jude Medical Tendril ${ }^{\circledR}$ \#2088-TC) and quadripolar left ventricular lead (St. Jude Medical Quartet $\left.{ }^{\mathrm{TM}} \# 1458-\mathrm{Q}\right)$ inserted via the coronary sinus. Satisfactory measurements were obtained in both implanted leads. Right ventricular lead was not implanted due to the presence of bioprosthetic tricuspid valve. During postimplantation visit, satisfactory measurements were once again obtained and the LVCap ${ }^{\mathrm{TM}}$ Confirm feature (St. Jude Medical) was evaluated for possible use. Despite obvious lack of ventricular capture, programming this feature on was recommended. Why was this algorithm's activation recommended despite the absence of ventricular capture? Can the LVCap ${ }^{\mathrm{TM}}$ Confirm feature be safely programmed on in this patient?

\section{Discussion}

This is the author's manuscript of the article published in final edited form as:

Kalra, V., Foreman, L., \& Bhakta, D. (2016). Apparent Failure of Automated Threshold Evaluation: What is the Mechanism? Pacing and Clinical Electrophysiology, 39(8), 870-872. https://doi.org/10.1111/pace.12865 
When performed manually, the LVCap ${ }^{\mathrm{TM}}$ Confirm setup (St. Jude Medical, St. Paul, MN, USA) determines its suitability for use prior to its activation. During the setup procedure, pacing pulses are delivered using the programmed LV pacing configuration at progressively declining output beginning at 4.5 $\mathrm{V}$ down to $0.25 \mathrm{~V}$. With each pulse, the evoked response is measured from the electrode serving as the cathode (in this example, the second/middle electrode from the tip of the quadripolar lead, or M2) and the generator can, to confirm successful ventricular capture or lack thereof, and to assess for noise that may be mistaken for capture. Each left ventricular (LV) pacing pulse is normally followed by a nearly simultaneous backup/“rescue” pulse via the right ventricular (RV) lead (which of course is absent in this patient) to prevent loss of capture (LOC) by a subthreshold LV stimulus and to avoid phrenic nerve stimulation with high output "rescue” LV pacing. During this patient's LVCap ${ }^{\mathrm{TM}}$ Confirm setup shown in Figure 1, the final four LV pulses at $0.25 \mathrm{~V}$ output are seen, obviously below the LV capture threshold, resulting in absence of ventricular capture, with essentially simultaneous though ineffectual backup pulses delivered through the nonexistent RV lead. Noise and stimulation artifact are seen in the (nonfunctional) RV recording channel. An LOC marker is not seen as this label is only displayed during a threshold test, not during setup. Regardless of these findings, the use of LVCap ${ }^{\mathrm{TM}}$ Confirm is recommended (horizontal solid arrow), apparently indicating satisfactory evoked response detection and noise rejection, even in light of lack of ventricular capture with backup RV pacing. The asterisk marks fusion of the sinus P wave and QRS complex, the latter originating from junctional escape rhythm in the presence of complete atrioventricular block.

Figure 2 demonstrates normal functioning of the LVCap ${ }^{\mathrm{TM}}$ Confirm setup from a different patient with St. Jude Medical Unify Assura ${ }^{\mathrm{TM}}$ 3357-40C biventricular implantable cardioverter defibrillator system. Here, three LV pulses at $0.5 \mathrm{~V}$ output are seen followed by the final four stimuli at $0.25 \mathrm{~V}$. In this example, absence of ventricular capture is not observed as backup pulses through the normal functioning RV lead are delivered. In this pacing-dependent patient, however, the LVCap ${ }^{\mathrm{TM}}$ Confirm feature is not 
recommended for use (horizontal solid arrow). The asterisks indicate fusion beats originating from nearly simultaneous premature ventricular complexes and ventricular paced beats.

Automatically adjusted pacing outputs have gained widespread popularity and have become standard features in pacing systems. A pacemaker's capability to measure pacing threshold and adjust its output accordingly has significant diagnostic and therapeutic implications. The ability to review measured threshold data may reveal trends signaling potential lead problems. More importantly, these features enhance pacing safety by assuring capture and allowing reduction in pacemaker battery consumption, consequently extending pacemaker longevity. [1, 2]

The presence of a permanent transvenous ventricular pacing lead has been associated with worsening tricuspid regurgitation when such leads traverse both native tricuspid valves as well as surgically repaired valves using annuloplasty rings.[3, 4] In our index patient, the presence of tricuspid valve bioprosthesis resulted in our decision to implant a pacing system incorporating only atrial and LV leads without an RV lead, to possibly prevent premature prosthetic valve regurgitation and degeneration. This approach of endovascular LV pacing via the coronary sinus without an RV lead has been reported with satisfactory results. $[5,6]$

While offering substantially more pacing options, the use of a quadripolar LV lead mandated the use of a quadripolar-compatible biventricular pacemaker pulse generator. This particular pacemaker model only allowed for safe operation of automatic LV output adjustment with the concomitant presence of an RV lead as described above, to avoid loss of ventricular capture. While a relatively uncommon scenario, the LVCap ${ }^{\text {TM }}$ Confirm algorithm should be avoided whenever problematic RV lead behavior is observed or when RV lead is altogether absent, of special importance in patients with pacing dependence. In this patient, further attempts to activate this feature were ultimately abandoned and fixed LV pacing output was programmed. 
Modern pacing systems have evolved to include a plethora of features enhancing their safety, longevity, programmability, and diagnostic capabilities rendering detailed knowledge of each system's functioning nearly impossible. However, as seen here, familiarity with a particular device's advantages, limitations, and potential adverse impact on a specific patient substrate are required to safely manage recipients of these increasingly complex systems. 


\section{References}

1. Clarke M, Liu B, Schüller H, Binner L, Kennergren C, Guerola M, Weinmann P, et al. Automatic adjustment of pacemaker stimulation output correlated with continuously monitored capture thresholds: A multicenter study. Pacing Clin Electrophysiol 1998; 21:1567-1575.

2. Lau C, Cameron DA, Nishimura SG, Ahern T, Freedman RA, Ellenbogen K, Greenberg S, et al. A cardiac evoked response algorithm providing threshold tracking: A North American multicenter study. Pacing Clin Electrophysiol 2000; 23:953-959.

3. Kim JB, Spevack DM, Tunick PA, Bullinga JR, Kronzon I, Chinitz LA, Reynolds HR. The effect of transvenous pacemaker and implantable cardioverter defibrillator lead placement on tricuspid valve function: An observational study. J Am Soc Echocardiogr 2008; 21:284-287.

4. McCarthy PM, Bhudia SK, Rajeswaran J, Hoercher KJ, Lytle BW, Cosgrove DM, Blackstone EH. Tricuspid valve repair: Durability and risk factors for failure. J Thorac Cardiovasc Surg 2004; 127:674-685.

5. Daubert JC, Ritter P, Le Breton H, Gras D, Leclerq C, Lazarus A, Mugica J, et al. Permanent left ventricular pacing with transvenous leads inserted into the coronary veins. Pacing Clin Electrophysiol 1998; 21:239-245.

6. Boriani G, Gardini B, Diemberger I, Bacchi Reggiani ML, Martignani C, Ziacchi M, Valzania C, et al. Meta-analysis of randomized controlled trials evaluating left ventricular vs. biventricular pacing in heart failure: Effect on all-cause mortality and hospitalizations. Eur J Heart Fail 2012; 14:652-660. 
Figure 1. Abnormal operation of LVCap ${ }^{\mathrm{TM}}$ Confirm setup from index patient in whom RV lead was absent. See text for details. $\mathrm{LV}=$ left ventricular; RV = right ventricular.

\section{Test Results: LVCap ${ }^{T M}$ Confirm Setup}

Recommended @ $0.8 \mathrm{~ms}$ (M2 - P4)

$1.75 \mathrm{~V} @ 1.2 \mathrm{~ms}(\mathrm{M} 3 \cdot \mathrm{P} 4)$
Aug 5, 2015 Jul 14, 2015

LVCap ${ }^{\mathrm{TM}}$ Confirm recommended

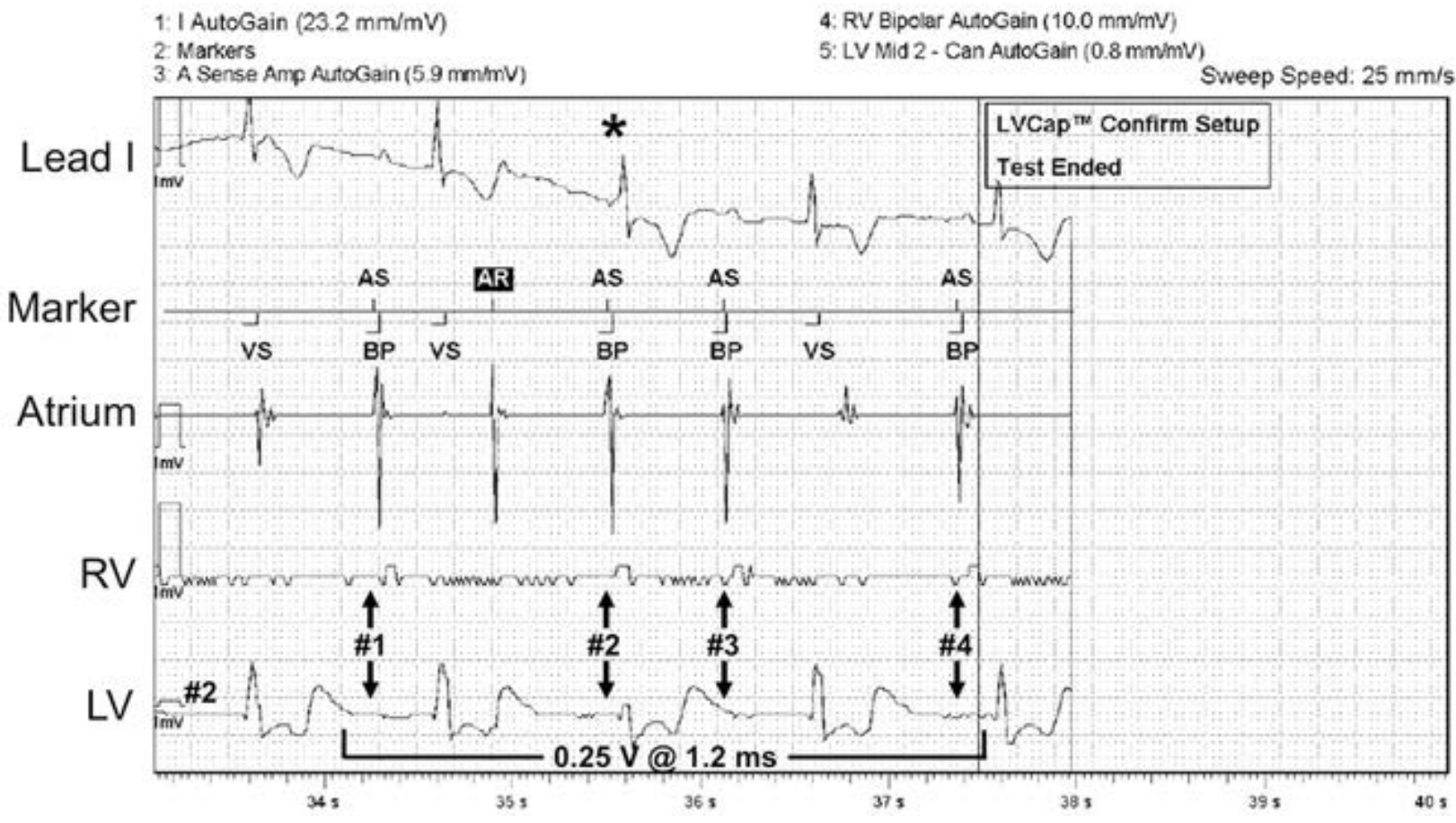


Figure 2. Normal operation of LVCap ${ }^{\mathrm{TM}}$ Confirm setup from a separate patient in whom normal functioning RV lead was present. See text for details. LV = left ventricular; RV = right ventricular.

\section{Test Results: LVCap ${ }^{\mathrm{TM}}$ Confirm Setup}

Pege 1 of 1

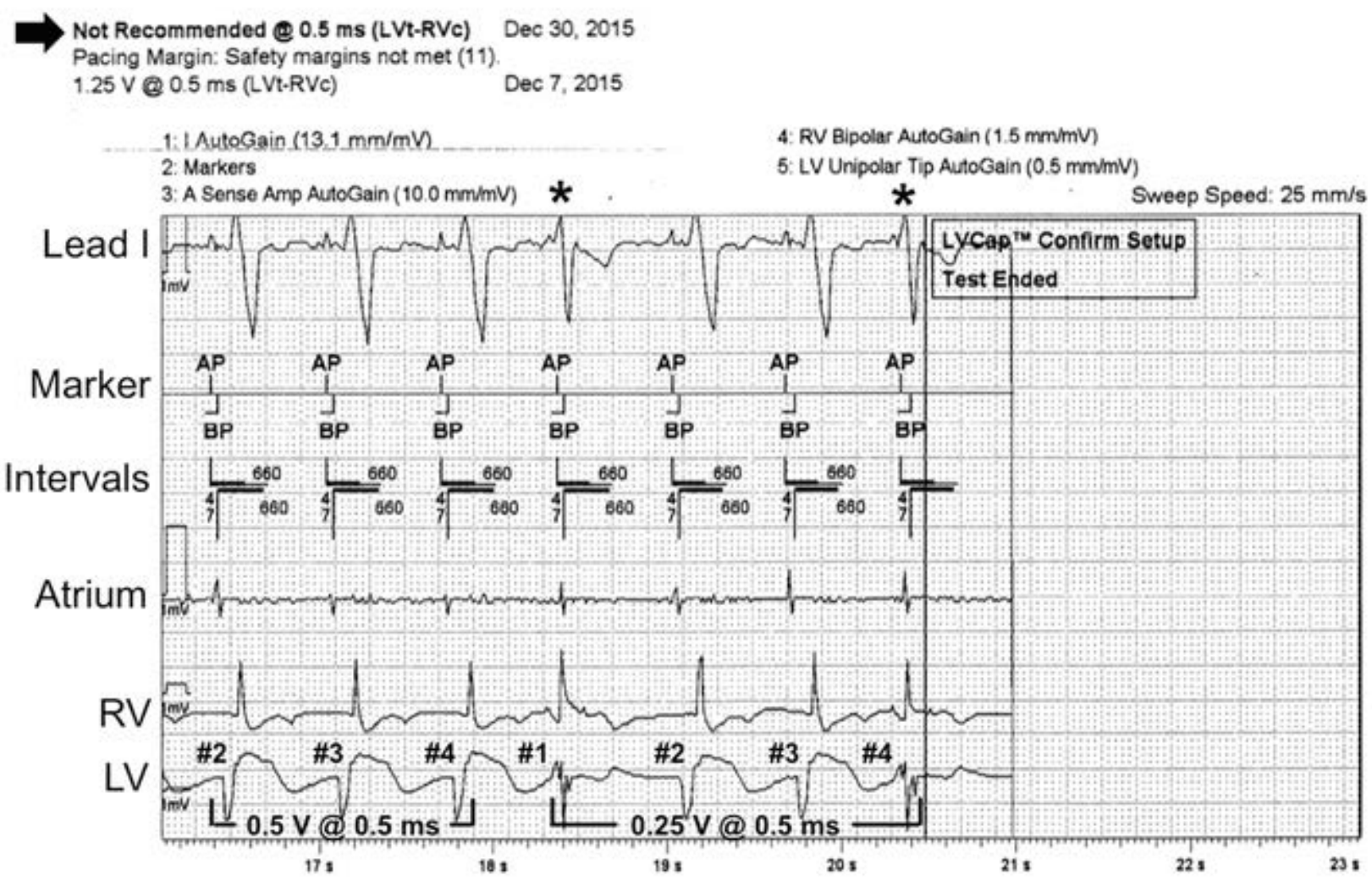

